\title{
SIGNIFICANCE OF CLINICAL AND LABORATORY MARKERS IN DISORDERS OF THE FUNCTIONAL CONDITION OF THE HEPATOBILIARY SYSTEM IN NEONATAL JAUNDICE
}

\author{
Yuliia Volosivska $^{1}$, Yuliia Hodovanets ${ }^{2}$, Lilia Yurieva ${ }^{3}$
}

\begin{abstract}
:
INTRODUCTION: The liver is the central organ of metabolic activity in the body.

OBJECTIVES: The purpose of this study was to determine predictors, major clinical features, and laboratory criteria for the severity of disorders of the functional state of the hepatobiliary system in newborns with neonatal jaundice (NJ). The study included 164 full-term infants. The main group - 92 children, with manifestations of pathological jaundice, was divided into two subgroups, depending on the severity of the pathology, considering concomitant perinatal pathology. IA group (46 children) includes children, condition of which was defined as moderate, IB (46 children) - children, condition of which was defined as severe, the control group consisted of 72 healthy term infants.
\end{abstract}

METHODS: The complex of examination methods includes: collection of anamnesis of antenatal and perinatal periods in the mother, clinical examination of the newborn and laboratory methods of examination: blood biochemical analysis (TSB blood, bilirubin fractions, activity of ALT enzymes, AST, ALP , $\gamma$-GT, glucose level of blood serum, cholesterol, triglycerides) ultrasound examination of infants' liver.

RESULTS: Clinical symptoms that confirmed the functional impairment of the hepatobiliary system (HBS) in newborns who had signs of $\mathrm{NJ}$ in perinatal pathology are: hepatosplenomegaly, impaired bowel function, hypoglycemia, anemic syndrome, hemorrhagic disorders. The results of biochemical studies of the blood showed an increase in the severity of the pathology, an increase in the level of TSB and the fraction of indirect bilirubin, activation of the cytolysis markers of ALT and AST, increase of LDH activity, decrease in the activity of $\gamma$-GT and ALP, as well as decrease in the level of total protein, albumin, glucose, increase in the level of urea, cholesterol and triglycerides. The most explicit manifestations of HBS dysfunction were found in newborns with a severe condition at birth and in the early neonatal period caused both by the $\mathrm{NJ}$ and other comorbidities. Taking into account the revealed deepening of biochemical changes in indicators of blood serum in correlation with the increase of clinical manifestations of pathology, the diagnosis of changes in the early stages will allow to diagnose in time the disorders of the functional state of HBS in NJ for the purpose of appropriate therapeutic correction.

CONCLUSION: The results of the analysis of anamnestic and clinical-laboratory parameters in newborns with NJ showed on the background of a clinical signs of dysfunction of the hepatobiliary system, the presence of significant biochemical changes, especially enzymatic activity, including, ALT, AST, LDH, ALP, and $\gamma$-GT and, also an increase in TBS levels and an indirect bilirubin fraction, a decrease in total protein and albumin, glucose, a rise in urea, cholesterol and triglycerides.

The use of a biochemical markers in newborns with clinical manifestations of neonatal jaundice, especially in cases of significant severity of the condition with concomitant perinatal pathology, requires more detailed analysis in order to determine the main pathogenesis of disorders of the functional state of the hepatobiliary system for the purpose of timely correction and improvement of the outcome of treatment.

UDC Classification: 616.36-008.5/.6-053.1-071-074, DOI: https://doi.org/10.12955/pmp.v1.108

Keywords: newborn, jaundice, liver, hepatobiliary system, biochemical markers

\section{Introduction}

In the process of complex diagnostics of the functional activity of an organism it is difficult to define an isolated disorder, considering the multifactorial changes of certain laboratory parameters. However, additional methods of investigation of disorders of the functional state of each individual organ system, in particular the hepatobiliary system, allows to identify the main pathological syndromes of dysfunction that determine the severity of the pathological process. It helps to correct properly the treatment for stabilization of disturbances of homeostasis of an organism and, accordingly, to reduce the clinical manifestations of organ's system dysfunction and, in general, clinical signs of pathology. The main factors of disorders of the functional state of the hepatobiliary system in newborns in neonatal jaundice are hypoxia and / or ischemia in the perinatal period (Singh et al., 2019, Birrer et al., 2007, Dhanjal et al., 2016).

\footnotetext{
${ }^{1}$ Higher state educational establishment of Ukraine "Bukovinian state medical university", Department of Pediatrics, Neonatology and Perinatal Medicine, Chernivtsi, Ukraine, volosivska@ukr.net

${ }^{2}$ Higher state educational establishment of Ukraine "Bukovinian state medical university", Department of Pediatrics, Neonatology and Perinatal Medicine, Chernivtsi, Ukraine, yul.godovanets@gmail.com

${ }^{3}$ Higher state educational establishment of Ukraine "Bukovinian state medical university", Department of Obstetrics, Gynecology and Perinatology, Chernivtsi, Ukraine, yurieva.lilia@bsmu.edu.ua
} 
The cytoplasm and hepatocyte organelles have many enzymes that are constantly involved in biochemical metabolic processes directed in to maintaining of homeostasis. In general, the liver performs more than 500 functions (Patel and Preedy 2017). The most researched indicators of functional activity of hepatocytes are: the bilirubin level (with fractions), enzyme activity: alanine aminotransferase (ALT), aspartate aminotransferase (AST), lactate dehydrogenase (LDH), gammaglutamyl transferase ( $\gamma$-GT) and alkaline phosphatase (ALP). These indicators are widely used in models of forecasting of liver dysfunction in children with any pathology (Zahmatkeshan et al., 2019, Thomson et al., 2009).

According to the literature, the following syndromes of pathogenesis of hepatobiliary system (HBS) dysfunction are revealed: cytolysis syndrome, markers of which are increases of enzymes activity of the ALT, AST, LDH, $\gamma$-GT, succinate dehydrogenase (SDH) (Patel and Preedy, 2017); cholestasis syndrome, markers of which are increases in ALP, $\gamma$-GT, 5-nucleotidase, and total serum bilirubin (TSB) (Patel and Preedy, 2017), mesenchymal-inflammatory reaction syndrome, as evidenced by serum immunoglobulins, as well as hepatocellular insufficiency syndrome, markers of which are levels of serum albumin, ceruloplasmin, and coagulation (Patel and Preedy, 2019).

The peculiarity of the liver acinus structure causes the increased sensitivity of the centrilobular zone to such factors as hypoxia, ischemia, viral damage. The increased levels of TSB affect's on to the rheological properties of bile (Papazovska Cherepnalkovski et al., 2015) increasing its viscosity, and, also the direct toxic effect of bilirubin on the membrane of hepatocytes and cell mitochondria is under discussing. The effect of parenteral nutrition on the development of cholestatic syndrome has been proven, while the late beginning of parenteral therapy is contributing to a higher serum bilirubin level (Jenniskens et al., 2018).

The issue of the functional state of the hepatobiliary system in newborns and young children received considerable attention in recent years (Torre, 2017; Chee et al., 2018; Khurshid et al., 2018). Many domestic and foreign authors emphasized the role of oxidative stress in the formation of pathology of organ systems in perinatal pathology, in particular the hepatobiliary system (Anil Batta, 2017).

In spite of the numerous studies that are being conducted, the degree of impaired functional status of the hepatobiliary system in hyperbilirubinemia, correlating with the severity of neonatal jaundice and gestational age of the baby, remains unclear. The problems of cellular and molecular mechanisms of liver dysfunction in neonatal jaundice, aspects of the prediction and differential diagnosis of the severity of the disorders, as well as the mechanisms that lead to insufficient conjugation of bilirubin and cause the pathology are still poorly researched. It remains uncertain whether these disorders are a decisive factor in the formation of chronic gastroenterological diseases in the later years (Shutova and Bahatska, 2017). Despite the increasing of knowledge about the etiology of diseases of hepatobiliary system, there isn't enough scientific researches on epidemiology and aspects of the emergence and progression of pathology in young children.

\section{Objective}

To define the risk factors for development, clinical and laboratory criteria for the severity of disorders of the functional state of the hepatobiliary system in neonatal jaundice of newborns.

\section{Materials and methods}

The main study group consisted of 92 full-term newborns (gestational age 37-41 weeks), which in the complex of clinical signs of perinatal pathology had clinical manifestations of neonatal jaundice. According to severity of general condition, children were divided into two groups: IA group (46 children) included newborns who had moderate condition's severity; IB group (46 children) - who had a severe course of perinatal pathology. The control group for comparison of results of additional methods of research consisted of 72 healthy full-term newborns. The total number of surveyed children involved in the study was 164 children.

The exclusion criteria for the creation of the main study group was congenital organic pathology of the hepatobiliary system and septic conditions of the newborn.

The complex of clinical and laboratory methods includes: collection of anamnesis of antenatal and perinatal periods in the mother, clinical examination of the newborn and laboratory methods of examination: blood biochemical analysis (TSB blood, bilirubin fractions, activity of ALT enzymes, AST, ALP , $\gamma$-GT, glucose level of blood serum) ultrasound examination of infants' liver. 
The complex of biochemical studies of newborn's blood serum was performed using an analyzer "ULTRA" of "Kone" (Finland, reagents of the firm) and apparatus for electrophoresis "Bekman" (Austria, reagents of the firm) in the maternity's hospital biochemical laboratory.

The examination of newborns was conducted in accordance with the basic provisions of GoodClinicalPractice (1996), the Council of Europe Convention on Human Rights and Biomedicine (1997), the World Health Association's Declaration on Ethical Principles for Scientific and Human Research (1964-2008). ), Order of the Ministry of Health of Ukraine No. 690 of September 23, 2009 (as amended in accordance with the Order of the Ministry of Health of Ukraine No. 523 of July 12, 2012) and approved by the Commission for Biomedical Ethics of the Higher State Educational Institution of Ukraine «Bukovinian State Medical University".

For reliable analysis of identified risk factors, data were evaluated using Statitica, 2010, MedCalc software (https://www.medcalc.org/index.php).

The odds ratio (OR), its standard error, and the 95\% confidence interval were calculated by Altman, 1991. Indicator $\mathrm{p}<0.05$ was considered as statistically significant.

The dissertation work is carried out within the Research Department of the Department of Pediatrics, Neonatology and Perinatal Medicine of the Higher state educational establishment of Ukraine "Bukovinian state medical university" on the theme: «Improvement of the directions of prognosis, diagnosis and treatment of perinatal pathology in newborns and young children, optimization of the schemes of follow-up observation and rehabilitation». State registration number 0115U002768, due date 01/01/2015 - 31/12/2019).

\section{Results of the study and their discussion.}

Analysis of the gestational period in mothers showed the presence of concomitant extragenital pathology in the vast majority of cases in main group of observations, including anemia, respectively (19 and 41.30\%), diffuse non-toxic goiter (14 and 30.43\%), kidney pathology (8 and $17.39 \%$ ), chronic cholecystitis (6 and 13.04\%), vascular dystonia (21 and 41.65\%). Gestational edema was observed in 10 and $21.74 \%$, and isoimmunization in 36 and $78.26 \%$. The vast majority of cases of obstetric pathology in mothers of group IB were represented by: placental dysfunction (17.39\%), premature rupture of fetal membranes $(19.57 \%)$, threat of abortion $(19.57 \%)$, polyhydramnios (6.34\%), and preeclampsia (8.69\%).

Assessment of anthropometric and sex differences of children at birth showed no significant differences according to the established groups:

Anthropometric indicators: IA group: body weight $3320 \pm 441 \mathrm{~g}$, length - $53 \pm 2.37 \mathrm{~cm}$., Head circumference $-34 \pm 2.01 \mathrm{~cm}$., Breast circumference $-33 \pm 2.2 \mathrm{~cm}$; IB group: body weight $3383 \pm 443 \mathrm{~g}$, length $-53 \pm 24.8 \mathrm{~cm}$. , head circumference $-34 \pm 2.1$; circumference of the chest $-35 \pm 2.6 \mathrm{~cm}$. control group: $(3333 \pm 433 \mathrm{~g}$., length $-53.11 \pm 2.67 \mathrm{~cm}$., head circumference $-34 \pm 2.01 \mathrm{~cm}$., chest circumference $33 \pm 2.07 \mathrm{~cm}$.

IA group: the percentage of male was $63.04 \%$; IB group - $56.52 \%$; control group: $50 \%$ of male. Female respectively $-39.13 \% ; 43,48 \% ; 50 \%$.

Evaluation of early neonatal adaptation on the Apgar scale for $1 \mathrm{~min}$. in children of IA group was estimated at $7 \pm 0.79$ per $1 \mathrm{~min}$. and $8 \pm 0.77$ for $5 \mathrm{~min}$., adaptation of children of IB group $-7 \pm 1.3$ per 1 min; and $8 \pm 1.08$ for $5 \mathrm{~min}$; The assessment of the control group children was $7 \pm 0.77$ and $8 \pm 0.56$ points, respectively.

The vast majority of children of the IA group - 62 of children (86.1\%) were exclusively on breastfeeding; group IB - only 4 children $(8.69 \%)$ received exclusively breastfeeding the rest - 42 children $(91.3 \%)$ were on mixed feeding, that was caused by the babies staying on the intensive care, nonnutritive sucking and the need of using of infusion therapy and phototherapy as the initial method of treating of jaundice in this group of children. Infusion therapy required $40(86.96 \%)$ children in group I and $2(4.34 \%)$ children in group I $(\mathrm{p}<0.0001)$. In order to identify the risk factors and to analyze the features of neonatal jaundice, the following factors were analyzed: isosensitization by $\mathrm{ABO}$ or rhesus system, the time of jaundice appearance, the presence of concomitant risk factors for pathology. 
Analysis of pregnancy and childbirth in mothers of observation groups showed that 6 children (13.04\%) from the IB group versus 2 children $(2.78 \%)$ in the control group (OR-5.25; 95\% CI: 1.01 27.25; z statistic- 1.97; $\mathrm{p}=0.048$ ) suffered from distress during the labor. In IA group fetal distress was in $2(4.34 \%)$ children, which didn't have a statistical significance compared to control (OR-1.59; 95\% CI: $0.22-11.70 ; \mathrm{z}$ statistical-0.46; $\mathrm{p}=0.65)$. Childbirth weakness was more likely to be present in the IB group - $12(26.08 \%)$ versus 7 (9.72\%) in the control group (OR-3.28; 95\% CI: 1.18-9.09; z statistic-2 , 28; $\mathrm{p}=0.022)$; in IA group $10(21.74 \%)$, which compared to the control group didn't have a statistical significance (OR- 2.58; 95\% CI: 0.90-7.36; z statistical-1.77; $\mathrm{p}=0.076$ ). Vacuum extraction of fetal and cephalogematoma in the IA group - $1(2.17 \%)$ and $2(4.35 \%)$ cases; children of the IB group - cephalogematoma and vacuum extraction occurred at the same frequency of 3 cases $(6.52 \%)$. control group $-2(2.78 \%)$ cases of vacuum extraction and 3 cases $(4.17 \%)$ of cephalogemate. There was no statistical significance compared to the control ( $\mathrm{p}<0.33, \mathrm{p}<0.57$ ).

A contributing factor in impaired hepatobiliary function in children of the IB group was probably the perinatal effect of hypoxia (diagnosis of asphyxia at birth had $4(8.67 \%)$ children in this group (1 of them was due to syndrome of meconium aspiration); $(8.67 \%)$ of the child, in $1(2.17 \%)$ case the syndrome of multiple organ failure developed.

Respiratory failures were observed in 10 children $(21.74 \%)$ of the IB group (severe in 4 children $(8.69 \%)$, versus $3(6.52 \%)$ in the group IA $(p<0.053)$. The quantity of children diagnosed with Newborn Hemolytic Disease ABO-conflict was 20 (43.48\%) and 16 (34.78\%) Rh according to $\mathrm{Rh}$ factor in group IB, in group IA group - $24(52.17 \%)$ and $9(19.56 \%)$ respectively. Operations on the exchange blood transfusion (EBT) needed $13(28.26 \%)$ children of group IB (2 children of which needed reopening of EBT). 54 children $(75.0 \%)$ in the control group had a diagnosis of CHD at childbirth, but the pathological jaundice hadn't developed in any case. It was highlighted that the appearance of jaundice on the 1-st day was significantly more common among the children of the IB group - 34 (73.91\%), compared to $13(28.26 \%)$ in IA group ( $\mathrm{p}<0.0001)$.

In order to establish the pathogenic condition of jaundice, the following risk factors were analyzed: isoimmunization by $\mathrm{ABO}$ or Rhesus system, time of appearance of jaundice, presence of concomitant pathology, intranasal complications (vacuum-extraction of the fetus, labor initiation with oxytocin, cephalogematoma).

Occurrence of jaundice on the 1 day was significantly more frequent among children of the IB group $34(73.91 \%)$, versus $13(28.26 \%)$ in group IA ( $\mathrm{p}<0.0001)$. On the other hand, in the IA group, the appearance of jaundice more often corresponded to II-III days of life 33 (71.74\%) versus $12(26.08 \%)$ in the group IB ( $\mathrm{p}<0.0001)$. 6 children $(13.04 \%)$ from the IB group suffered from distress during childbirth versus 2 children $(2.78 \%)$ in the control group ( $\mathrm{p}<0.058)$. Childbirth weakness was more likely to be present in children of the IA group - $12(26.08 \%)$ (p <0.023) compared to the control group, in the IB group $10(21.74 \%)$ versus $7(9.72 \%)$ in the control group (p <0.077). Vacuum fetal extraction and cephalogematoma were present with the same frequency of $3(6.52 \%)$ in children of IB group and had no statistical significance compared to the control group ( $\mathrm{p}<0.33$, $\mathrm{p}<0.57$ ). 4 children (8.69\%) from the IB group in delivery room needed a conventional mechanical ventilation. Respiratory failures were observed in 10 children $(21.74 \%)$ of the IB group (severe in 4 children $(8.69 \%)$ ), versus $3(6.52 \%)$ in the IA group $(\mathrm{p}<0.053)$. The frequency of isoimmunization by the $\mathrm{ABO}$ system was $20(43.48 \%)$, according to the Rh factor - 16 (34.78\%) in IB group, in IA group 24 (52.17\%) and 9 (19.56\%), respectively. In the control group, 54 children (75\%) had a diagnosis of risk of Hemolytic Disease at birth, but the pathological jaundice had not developed in any case.

The most common clinical symptoms observed in newborns in the perinatal pathology group were: hepatosplenomegaly: 7 (15.22\%) in group IB; 1 (2.17\%) in group IA OR- 8.08; 95\%CI:0.95-68.56; z statistic-1.91; $\mathrm{p}=0.056)$ in the control group $1(1.39 \%)$; intestinal dysfunction, more explicit in children of the IB group - 7 (15.22\%) compared to 4 (8.69\%) in group IA (OR-1.88; 95\%CI:0.516.93; z statistic-0.95; $\mathrm{p}=0.34)$; hypoglycemia - in 7(15.22\%) group IB та and $1(2.17 \%)$ group IA (OR- 8.08; 95\%CI:0.95-68.56; z statistic-1.91; $\mathrm{p}=0.056)$; anemic syndrome - in $9(19.57 \%)$ with severe condition in full-term newborns (IB group) and in 1 (2.17\%) with moderate severity (IA group). Anemic syndrome - in $9(19.57 \%)$ with severe condition in term infants (IB group) and in 1 $(2.17 \%)$ with moderate severity (IA group) (OR-10.94; 95\% CI: $1,33-90.40$; z statistic-2.22; p = 
0.026). Haemorrhagic disorders were reported in 5 children $(10.87 \%)$ of the group IV, which, in our opinion, was caused to some extent by impaired liver synthetic function.

\begin{tabular}{|c|c|c|c|c|c|c|}
\hline \multirow{3}{*}{ Parameters } & \multicolumn{6}{|c|}{ Observation groups } \\
\hline & \multicolumn{2}{|c|}{$\begin{array}{l}\text { II group } \\
\text { N (72) }\end{array}$} & \multicolumn{2}{|c|}{$\begin{array}{l}\text { IA group } \\
\mathbf{N}(46)\end{array}$} & \multicolumn{2}{|c|}{$\begin{array}{l}\text { IB group } \\
N(46)\end{array}$} \\
\hline & $\mathbf{M} \pm \mathbf{m}$ & min-max & $\mathbf{M} \pm \mathbf{m}$ & $\min -\max$ & $\mathbf{M} \pm \mathbf{m}$ & min-max \\
\hline $\begin{array}{l}\text { TSB level of } \\
\text { umbilical blood } \\
(\mu \mathrm{mol} / \mathrm{L})\end{array}$ & 32.3 & $27.9-34.8$ & $38.46 \pm 1.49$ & $34.12-44.9$ & $56.24 \pm 2.75$ & $34.66-117$ \\
\hline $\begin{array}{l}\text { Maximum } \\
\text { blood's level } \\
\text { TSB on the } 1 \\
\text { day }(\mu \mathrm{mol} / \mathrm{L})\end{array}$ & - & & $69.29 \pm 3.5$ & $34.92-74.54$ & $140.69 \pm 7.6$ & $93.12-271.49$ \\
\hline $\begin{array}{l}\text { Maximum } \\
\text { blood's level } \\
\text { TSB on the } 3 \text { day } \\
(\mu \mathrm{mol} / \mathrm{L})\end{array}$ & - & & $268.45 \pm 12.56$ & $\begin{array}{l}162.96- \\
291.9\end{array}$ & $264.6 \pm 13.4$ & $\begin{array}{l}106.27- \\
390.07\end{array}$ \\
\hline $\begin{array}{l}\text { Conjugated } \\
(\mu \mathrm{mol} / \mathrm{L})\end{array}$ & $7.3 \pm 0.29$ & $1.76-12.02$ & $7.2 \pm 0.49$ & $6.28-8.08$ & $\begin{array}{c}12.58 \pm 5.11 \\
* \circ\end{array}$ & $1.78-21.12$ \\
\hline $\begin{array}{l}\text { Unconjugated } \\
(\mu \mathrm{mol} / \mathrm{L})\end{array}$ & $\begin{array}{c}159.68 \pm \\
58.78 \\
\end{array}$ & $\begin{array}{l}56.32- \\
118.54 \\
\end{array}$ & $233.78 \pm 20.14^{\circ}$ & $\begin{array}{l}194.28- \\
265.52 \\
\end{array}$ & $\begin{array}{c}201.14 \pm 38 . \\
68 * 0\end{array}$ & $80-374.09$ \\
\hline
\end{tabular}

The highest cord blood bilirubin concentration was observed in children of group IB $(56.24 \mu \mathrm{mol} / 1$ vs. $32.3 \mu \mathrm{mol} / 1$ in the control group $(\mathrm{p}<0.0001)$, this is partly because of the quantity of kids, whose severe conditions were caused by the hemolytic disease of the newborn within the perinatal pathology. Analysis of serum bilirubin growth showed a difference in the quantitative value of total serum bilirubin on the first day in children with IB and IA $(p<0.0001)$, and therefore the degree of loading on the functional activity of the liver under these conditions was greater in children of the IB group.

A positive development dynamics of TSB increased in maximum concentration on the 3-rd day was observed in children of the IA and IB groups (Table 1).

\begin{tabular}{|c|c|c|c|}
\hline \multirow{3}{*}{ Parameters } & \multicolumn{3}{|c|}{ Observation groups } \\
\hline & $\begin{array}{c}\text { II control group } \\
(n=72)\end{array}$ & $\begin{array}{c}\text { IA group } \\
(\mathrm{n}=46)\end{array}$ & $\begin{array}{c}\text { IB group } \\
(\mathrm{n}=46)\end{array}$ \\
\hline & $\mathbf{M} \pm \mathbf{m}$ & $\mathbf{M} \pm \mathbf{m}$ & $\mathbf{M} \pm \mathbf{m}$ \\
\hline Total protein (g/l) & $63.52 \pm 3.02$ & $57.48 \pm 2.58^{\circ}$ & $54.83 \pm 4.48^{\circ *}$ \\
\hline Albumin (g / l) & $34.56 \pm 1.07$ & $31.28 \pm 1.02^{\circ}$ & $23.82 \pm 1.18^{\circ *}$ \\
\hline ALT (units / I) & $16.54 \pm 1.45$ & $17.04 \pm 1.20$ & $28.42 \pm 3.69^{\circ *}$ \\
\hline AST (units / l) & $48.61 \pm 4.84$ & $50.80 \pm 3.04$ & $51.92 \pm 6.69^{\circ}$ \\
\hline$\gamma$-GT (units / I) & $90.8 \pm 8.71$ & $70.28 \pm 2.73^{\circ}$ & $74.55 \pm 3.16^{*}$ \\
\hline LDH (units / l) & $579.2 \pm 26.47$ & $575.04 \pm 19.46$ & $808.59 \pm 36.84 *$ \\
\hline ALP (units / l) & $277.0 \pm 17.74$ & $286.12 \pm 11.23^{\circ}$ & $192.50 \pm 7.51 *$ \\
\hline Glucose ( mmol / l) & $3.35 \pm 0.25$ & $3,88 \pm 0,36^{\circ}$ & $3.28 \pm 0.42 *$ \\
\hline Urea $(\mathrm{mmol} / \mathrm{l})$ & $3.2 \pm 0.16$ & $4.12 \pm 0.64^{\circ}$ & $3.45 \pm 0.75^{*}$ \\
\hline Cholesterol (mmol / l) & $1.7 \pm 0.1$ & $2.11 \pm 0.09^{\circ}$ & $2.02 \pm 0.095^{*}$ \\
\hline Triglycerides $(\mathrm{mmol} / \mathrm{l})$ & $0.6 \pm 0.003$ & $1.11 \pm 0.007^{\circ}$ & $0.75 \pm 0.067 *$ \\
\hline \multicolumn{4}{|c|}{$\begin{array}{l}* \text { - p value }<0.05 \text { compared with subgroup data. } \\
{ }^{\circ} \text { - p value }<0.05 \text { compared with the control group. }\end{array}$} \\
\hline \multicolumn{4}{|c|}{ Source: Volosivska (2020) } \\
\hline
\end{tabular}

The results of studies showed the tendency of total protein levels in children to deepen the deficit with increasing of severity of pathology $57.48 \mathrm{~g} / 1$ and $54.83 \mathrm{~g} / 1$ in groups IA and IB, respectively, in the control group (group II) this indicator was $63.52 \mathrm{~g} / 1$ ( $\mathrm{p}<0.05$ ) (Table 2). We think that these changes 
caused by the decrease of a liver synthesizing function and by the increase of a protein intake. A significant decrease in the albumin level - up to $23.82 \mathrm{~g} / 1$ was observed in newborns of the IB group, along with the fact that in the children of the IA group the value of the indicator corresponded to the lower limit of the norm (Table 2), that is probably caused by the increased use of albumin as a transport form for bilirubin. It's important that the presented changes of indicators, are also the consequence of the decrease of the synthetic function of the liver. So, this defines one of the links in the pathogenesis of the development of hemorrhagic syndrome in children who had significant severity of the condition (IB observation group). Hypoproteinemic syndrome also leads to a decrease in oncotic blood pressure and the development of edema.

Activation of ALT and AST cytolysis markers had a probable tendency to activation with the increasing severity of the pathology $(\mathrm{p}<0.05)$, however, we did not receive the critical exceedances of the age-related cytolysis rates observed for example in hepatitis, but this tendency may be due to the toxic effect of bilirubin itself on the hepatocyte membrane. It can also be important during discussing of the pathogenetic links of the onset of the severity of HBS dysfunction in the face of negative effects of hypoxia and ischemia on the baby's body at birth.

The activity of the $\gamma$-GT enzyme in the children of the observation groups tended to decrease compared to the control group, which probably indicate an inhibition of the glutathione detoxification system in the neonatal jaundice. A more explicit decreasing tendency was observed in newborns of the IB group who had more severe concomitant pathology. According to the literature $\gamma$-GT is a highly sensitive, membrane-specific enzyme involved in glutathione metabolism and amino acid resorption. Isolated increase of serum $\gamma$-GT is thought to be a sign of a hypoxic liver damage in the absence of concomitant increase in ALP activity (Dhanjal et al., 2016). The ALP activity in comparison to the indicators of the control group, tended to increase in children of the IA group, with a significant decrease in activity level of children in the IB group, which probably indicates the lack of a function of this enzyme in the severe condition of a newborn.

LDH 1,2 is an integral part of the lactate-pyruvate system that participates in the conversion of hydroxybutyrate, the final product of lipid breakdown, and provides the integration of carbohydrate and lipid metabolism. During the study, a significant increase of LDH was observed with increasing severity of the pathology, which confirm the significant increase in the number of children IB study group - up to $808.59 \pm 36.84 \mathrm{U} / 1$ compared with control indicators $-579.2 \pm 26.47 \mathrm{U} / 1$ (p <0.05) (Table 2). According to the data obtained, the activity of ALP significantly decreased, compared to the control group, in the severe condition of newborns - up to $192.50 \pm 7.51 \mathrm{U} / 1$ (Table 2). According to the literature, ALP is responsible for the dephosphorylation and release of glucose from tissues. It is also involved in the formation of a large amount of inorganic phosphate that significantly affects the bioenergy of cells in the body (Peters et al., 2015),(Pettengill et al., 2017).

Serum glucose levels increased significantly in the IA group compared to the control group, which can be explained by the nature of the stress response of the organism at the average severity of the condition of the newborn, and decreased in the severe condition, indicating a glucose metabolism disorder, one of the pathological links of which are changes in the metabolic activity of the liver under conditions of hyperbilirubinemia.

According to the literature, disorders of the fat metabolism, confirming the failure of the liver, include: disorders of bile formation and bile excretion; impaired synthesis of triglycerides, phospholipids, cholesterol, as well as the formation of blood plasma lipoproteins, which contributes to the formation of ketone bodies and liver steatosis (Biomarkers in Liver Disease, 2017). According to our data, the level of urea, cholesterol and triglycerides under conditions of hyperbilirubinemia tended to increase, compared to the control group, in children with moderate severity (IA group) and decrease in children with a severe condition (IB group), which indicates a metabolic disorder and, in on our opinion, needs further study to clarify the pathogenetic links of liver dysfunction, both by clinical and experimental methods.

\section{Conclusions}

1. The results of the analysis of anamnestic and clinical-laboratory parameters in newborns with NJ showed on the background of a clinical signs of dysfunction of the hepatobiliary system, the presence of significant biochemical changes, especially enzymatic activity, including, ALT, AST, LDH, ALP, 
and $\gamma$-GT and, also an increase in TBS levels and an indirect bilirubin fraction, a decrease in total protein and albumin, glucose, a rise in urea, cholesterol and triglycerides.

2. Using biochemical markers in newborns with clinical manifestations of neonatal jaundice, especially in cases of significant severity of the condition with concomitant perinatal pathology, requires more detailed analysis in order to determine the main pathogenesis of disorders of the functional state of the hepatobiliary system for the purpose of timely correction and improvement of the outcome of treatment.

Further researches perspective

Conducting a deep analysis of clinical and laboratory indicators characterizing hepatobiliary system dysfunction, to study the possibility of differential diagnosis of the severity of disorders at neonatal jaundice, taking into account the course of concomitant pathology.

\section{Conflict of interests}

The authors do not declare any conflict of interest.

\section{Acknowledgements}

This scientific research is performed within the framework of the research work of the Department of Pediatrics, Neonatology and Perinatal Medicine of the Higher State Educational Institution of Ukraine "Bukovinian State Medical University" on the theme: "Improvement of the directions of prognosis, diagnosis and treatment of perinatal pathology in newborns and children observation and rehabilitation" (state registration number 0115U002768) is an initiative based on self-financing.

\section{References}

Anil Batta, D. (2017). Bilirubin as savior of biological system. International Journal Of Current

Research In Medical Sciences, 3(2), 21-27. https://doi.org/10.22192/ijcrms.2017.03.02.004

Biomarkers in Liver Disease. (2017). https://doi.org/10.1007/978-94-007-7675-3

Birrer, R., Takuda, Y., \& Takara, T. (2007). Hypoxic Hepatopathy: Pathophysiology and Prognosis. Internal Medicine, 46(14), 1063-1070. https://doi.org/10.2169/internalmedicine.46.0059

Chee, Y., Chung, P., Wong, R., \& Wong, K. (2018). Jaundice in infants and children: causes, diagnosis and management. Hong Kong Medical Journal. https://doi.org/10.12809/hkmj187245

Dhanjal, G., Kaur, N., \& Kaur, H. (2016). Study of Liver Function Test in Perinatal Asphyxia at a Tertiary Care Center in Haryana. International Archives Of Biomedical And Clinical Research, 2(4). https://doi.org/10.21276/iabcr.2016.2.4.6

Jenniskens, M., Güiza, F., Haghedooren, R., Verbruggen, S., Joosten, K., Langouche, L., \& Van den Berghe, G. (2018). Prevalence and Prognostic Value of Abnormal Liver Test Results in Critically Ill Children and the Impact of Delaying Parenteral Nutrition*. Pediatric Critical Care Medicine, 19(12), 1120-1129. https://doi.org/10.1097/pcc.0000000000001734

Khurshid, F., \& Medves, J. (2018). Effectiveness of universal hyperbilirubinemia screening on newborn health. JBI Database Of Systematic Reviews And Implementation Reports, 16(2), 287-290. https://doi.org/10.11124/jbisrir-2016-003338

Papazovska Cherepnalkovski, A., Krzelj, V., Zafirovska-Ivanovska, B., Gruev, T., Markic, J., \& Aluloska, N. et al. (2015). Evaluation of Neonatal Hemolytic Jaundice: Clinical and Laboratory Parameters. Open Access Macedonian Journal Of Medical Sciences, 3(1), 694. doi: 10.3889/oamjms.2015.129

Patel, V., \& Preedy, V. (2019). Biomarkers in Liver Disease. Springer Netherlands.

Pettengill M, Matute JD, Tresenriter M, Hibbert J, Burgner D, Richmond P, et al. (2017) Human alkaline phosphatase dephosphorylates microbial products and is elevated in preterm neonates with a history of late-onset sepsis. PLoS ONE 12(4): e0175936. https://doi.org/10.1371/journal.pone.0175936

Shutova O.V., \& Bahatska N. V (2017). Otsinka endohennykh ta ekzohennykh faktoriv ryzyku formuvannia zhovchnokamianoi khvoroby v dytiachomu ta pidlitkovomu vitsi.[ Assessment of endogenous and exogenous risk factors for cholelithiasis in childhood and adolescence] Bulletin of problems of biology and medicine, 3 (4), 245-249. DOI 10.29254/2077-4214-2017-4-3-141-245-249

Singh,J.Poonia,A.K.,(2019).Liver Dysfunction in Perinatal Asphyxia, 7(3), PO06-PO08. https://www.doi.org/10.7860/JCDR/2019/41616/2253

Thomson, S., Cowan, M., Johnston, I., Musa, S., Grounds, M., \& Rahman, T. (2009). 'Liver function tests' on the intensive care unit: a prospective, observational study. Intensive Care Medicine, 35(8), 1406-1411. https://doi.org/10.1007/s00134009-1511-7

Torre G. (2017) Gastroenterology: Focus on Children with Liver Problems. In: Garganese M., D'Errico G. (eds) Conventional Nuclear Medicine in Pediatrics. Springer, Cham. https://doi.org/10.1007/978-3-319-43181-9_18

Zahmatkeshan, M., Serati, Z., Freydooni, S., Safarpour, A. R., Esmailnejad, A., \& Haghbin, S. (2019). Prediction of Early Liver Failure in Pediatric Patients Admitted to Intensive Care Unit. Middle East journal of digestive diseases, 11(3), 141146. doi:10.15171/mejdd.2019.140 